- Inequalities are found in community oral health - with affluent populations experiencing good oral health, whereas the converse is observed in deprived populations.

- The Department of Health would like to see dentists working with communities to address oral health inequalities.

- The new dental contract provides the mechanism to facilitate this.

\title{
Improving oral health with the new dental contract
}

\author{
W. Richards ${ }^{1}$ and A. Toy ${ }^{2}$
}

\begin{abstract}
The challenge for the twenty-first century for oral health is to reduce the social inequality observed in populations. This challenge has been made explicit by the Department of Health $(\mathrm{DH})$ in England. Turning rhetoric into reality is difficult for all concerned whatever field or profession. A cohesive model that embraces philosophical ideals can help individuals achieve change as it provides structure on which to develop ideas. This paper describes a model of care in general dental practice that fits the requirements of the $\mathrm{DoH}$ in addressing social inequalities, in the context of developing general dental services.
\end{abstract}

The Department of Health (DH) in England have recently released a CD-Rom entitled Improving oral health with the new dental contract. ${ }^{1}$ This has been distributed to general dental practitioners (GDPs) in England to help them develop their practices in such a way that will impact on the oral health of the populations they serve.

It is refreshing to see such an initiative from the $\mathrm{DH}$, on numerous accounts. Firstly, the Chief Dental Officer makes it explicit that the role of the dentist in the twenty-first century is not merely to concentrate on the 'one to one' aspect of patient care but to take account of the wider community. This is a fundamental change in perception of role for

\footnotetext{
1*Professor, Faculty of Health, Sport and Science, Uni-
versity of Glamorgan, Pontypridd CF37 1DL: ${ }^{2}$ General

${ }^{1 *}$ Professor, Faculty of Health, Sport and Science, Uni-
versity of Glamorgan, Pontypridd CF37 1DL; ${ }^{2}$ General Dental Practitioner, Gorse Covert Dental Practice, 34 Maxwell Drive, Loughborough, LE11 4RZ

${ }^{*}$ Correspondence to: Professor Wayne Richards Email:wrichard@glam.ac.uk
}

many dentists. This new role is put into context in the CD by the Chief Medical Officer who states "the NHS has moved from "just a sickness service" to a "promotion of health" service'.

Secondly, it is recognised that there have been no incentives for dentists and their teams to engage with the population beyond the surgery. This has now been addressed through the new dental contract by giving Primary Care Trusts (PCTs) the responsibility to provide incentives for services that promote health and demonstrate health outcomes.

Thirdly, the aspects of population health that will be seen as important for PCTs will be the inequalities observed in population health statistics, and this is highlighted by demonstrating Julian Tudor-Hart's Inverse care law in the distribution of dentists in relation to oral health. ${ }^{2}$ Therefore, in theory the PCTs will now support practices that impact on health inequalities in their local populations.

The conclusion from this is that dentists will need to 'provide new ways of working' and it is implied that if they do so they will be adequately rewarded by their PCTs. For this to work, PCTs will need the insight to recognise general dental practice models which 'provide new ways of working'.

This is where the laudable aims of the CD-Rom fail to materialise. The $\mathrm{DH}$ provides no coherent model or vision to inspire a practitioner to change to a 'new way' or give PCTs a clear lead. Instead, what is offered are details of evidencebased prevention along with some short video clips of seemingly unscripted interviews with members of the dental team. Each participant has an interesting comment to make and the facts are good to know - but it all seems a bit superficial. How does it all fit together? What are the underlying principles that are driving their actions? What sort of strategic planning do we need to do? Where is the practical guidance on personnel, financial and physical resources, to name but a few?

The message from this CD-Rom has the hallmarks of the 'hard, high ground' thinking of academia. Unfortunately, the academics do not always appreciate that GDPs are operating in the "swampy 


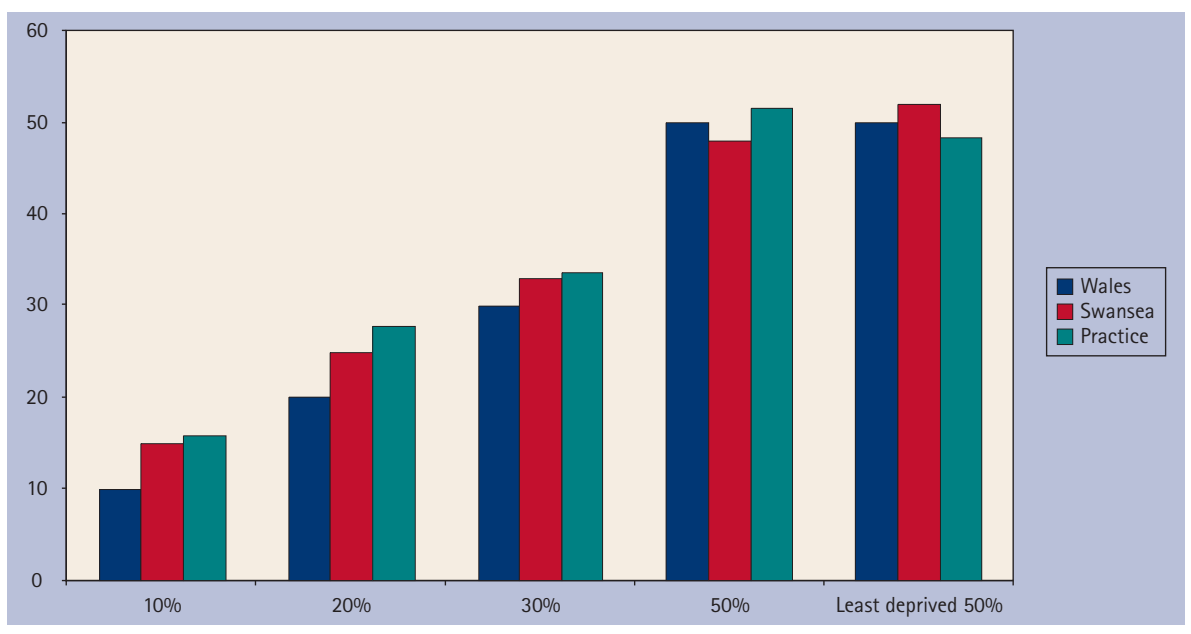

Fig. 1 Deprivation in Wales, Swansea and practice

lowlands'. ${ }^{3}$ Practice owners (including the corporates) are unlikely to take the substantial risk of revolutionising their practice unless they receive a strong lead from the PCTs who, in turn, are provided with a clear vision of the future from the Department of Health - a vision that is grounded in the reality of everyday practice.

We would like to offer a vision that has been the subject of some wet-fingered research in Wales. Community General Dental Practice (CGDP) is a model of practice that has already been documented in the literature. ${ }^{4,5}$ This model of practice has all the elements of 'good practice' identified in the CD-Rom. There are four innovative features to the CGDP model:

- Maintaining physical and social access to the whole community registration and continuing care

- Health focused philosophy prevention and oral health assessment for patients

- Total quality management development of the dental team

- Oral health-related quality of life measurement - objective measurement of patient experience.

The health outcomes demonstrating the above have also been reported in the literature. ${ }^{6-9}$ Clearly, maintaining physical and social access to the whole community is easily measured though 'notional list' size and deprivation profile. If the list size is the same or greater than the BDA adjusted dentist to population ratio and the deprivation profile reflects the community then the dentist is fulfilling the first element of CGDP. Historically the Dental Practice Board
(DPB) reported list size to each NHS dentist every month through registrations. Currently, no report is provided for the dentist as there is no 'registration' but 'notional list' size is reported to the PCT/ LHB (in Wales) through the number of unique individuals attending within a given time frame. At the time of writing the new contract has been in operation for one year. It should be noted here that if a patient attends every six months then that patient would only score as one unique individual within a two-year time frame (not four). Patient postcodes can be used to formulate practice deprivation profiles. Postcode data collected in 2006 were used to formulate the profile shown in Figure 1 using the Welsh Index of Multiple Deprivation (WIMD). The DPB have reported on the use of postcode analysis and therefore have the ability to analyse postcode data.

A written explicit health promotion protocol can demonstrate the second element. An explanation of the protocol used in one practice has been reported in the literature. ${ }^{10} \mathrm{~A}$ practice can devise its own protocol. The protocol should include the use of patient held documentation that records health status at examination.

The third element can be achieved through external accreditation by recognised Quality Assurance (QA) assessors, of which there are many. The QA procedures can include auditing the health promotion protocol by the assessor. The ethos of QA includes problem solving for the whole organisational team and therefore any problem/complaint becomes the domain of the dental team. As such future solutions/actions are owned by the individuals within the team, thus increasing the likelihood of compliance.

Finally, the objective measurement of patient experience can be achieved using Oral Health-Related Quality of Life (OHRQoL) measures. There are many OHRQoL measures available. Their use can be audited as a part of QA within a practice. The Subjective Oral Health Status Indicators (SOSHI) and also the short form of the Oral Health Impact Profile (OHIP) have been used in one location to fulfil the criteria of CGDP. The short form OHIP was also used in the Adult Dental Health Survey in 1998 and therefore can be used for useful comparisons.

This research only shows evidence of what has been possible by one dental team in one practice setting. However, as a model it could be a useful template for development. As it stands it does not provide an answer to the Welsh Assembly Government (WAG) or DH's problems. Further research is required to develop the model so that it may be applied in a variety of practice settings.

If the DH or WAG are truly interested in using GDPs to transform the oral health of the nation, then some effort is needed not just telling the $w h y$ but also the how this new service should be provided. That means translational research involving practitioners who understand the complexities of managing the resources of general dental practice. ${ }^{11}$ The new NHS dental contract has the potential to create a twenty-first century service that meets the needs of patients, tax payers and the profession. The DH and WAG need to enable the development of the evidence base that will give PCTs/LHBs and the dental profession a real world vision of this new NHS service. A service that is fit for purpose, value for money and that financially rewards dentists for the cultivation of oral health in the community.

\section{Concluding remarks}

Bloomfield in the early 1990s presented pathways for change for dentistry. ${ }^{12}$ Over 15 years later a new dental contract has been delivered. According to the DH's CD-Rom this new contract is the key to enable 'new ways of working'. Therefore, PCTs/LHBs are fundamental facilitators of change. Sadly, the LHB responsible for the area in which the above model has been developed, fail to recognise its existence. 
1. Department of Health. Improving oral health with the new dental contract. 2007.

2. Tudor-Hart J. The inverse care law. Lancet 1971; 1: 405-412.

3. Schon D. Educating the reflective practitioner: towards a new design for teaching and learning in the professions. San Francisco: Jossey-Bass, 1987.

4. Richards W Ameen J R M Coll A M. The community general dental practitioner. Br J Healthc Manage 2005: 11: 308-312.

5. Richards W Evans L. From rhetoric to reality: PPI in general dental practice. Br J Healthc Manage 2006;
12: $50-54$.

6. Richards W Scourfield S. Oral ill health in a general dental practice in South Wales. Prim Dent Care 1996; 3: 6-13

7. Higgs G Richards W. The use of Geographical Information Systems in examining variations in sociodemographic profiles of dental practice catchments: a case study of a Swansea practice. Prim Dent Care 2002; 9: 63-69

8. Richards W. Oral ill health and deprivation among patients of a general dental practice in South Wales. Prim Dent Care 2002; 9: 105-112.
9. Richards $W$, Ameen J. The impact of attendance patterns on oral health. Br Dent J 2002; 192: 697-702.

10. Richards W. Shifting the focus in dental care. In Riley C, Warner M, Pullen A, Piggot C et al. Releasing resource to achieve health gain. pp 124-131. Oxford, Radcliffe Medical Press, 1995.

11. Cooksey D. A review of UK health research funding. 2006. http://www.hm-treasury.gov.uk/media/4/A pbr06 cooksey final report 636.pdf.

12. Bloomfield K. Fundamental review of dental remuneration. 1992. 